\title{
Qualification of selective laser-melted Al alloys against fatigue damage by means of measurement and modeling techniques
}

\author{
Mustafa Awd $^{1 *}$, Jan Johannsen ${ }^{2}$, Shafaqat Siddique ${ }^{1}$, Claus Emmelmann ${ }^{2}$, and Frank Walther ${ }^{1}$ \\ ${ }^{1}$ Department of Materials Test Engineering (WPT), TU Dortmund University, D-44227, Dortmund, Germany \\ ${ }^{2}$ Fraunhofer Research Institute for Additive Manufacturing Technologies (IAPT), D-21029 Hamburg, Germany
}

\begin{abstract}
Aluminum alloys processed through selective laser melting possess unique features of microstructure, defect morphology and mechanical properties. Constitution of fine cellular dendrites results from the high-cooling rate of the melt pool during the consolidation process. Investigation of the microstructure by scanning electron microscopy identifies supersaturation of Si particles as a secondary strengthening mechanism. On the contrary, platform heating that induces coarser microstructure leads to migration of $\mathrm{Si}$ particles from the $\mathrm{Al}$ matrix to the eutectic phase. As a result, tensile strength is reduced by $\sim 3 \%$, while fracture strain is increased by $\sim 17 \%$. Fine-grained structures exhibit a lower amount of plastic damage accumulation as well as delayed crack initiation as determined by the applied measurement techniques. Finite element models of the investigated configurations are obtained using scans of computed tomography under consideration of process-induced defects. Comparison of modeling and experimental results concluded that dominant fatigue damage mechanisms are related to the loading regime from low-cycle (LCF) to very-high-cycle fatigue (VHCF). Thus, process-inherent features of microstructure and porosity have different quantitative effects concerning the applied load. In VHCF, a material configuration with platform heating possesses an improved fatigue strength by $\sim 33 \%$ at $1 \mathrm{E} 9$ cycles, concerning the material configuration without platform heating.
\end{abstract}

\section{Introduction}

Production of metallic components using layer-wise build-up in powder-bed-fusion processes, like selective laser melting (SLM), made the most significant progress from rapid prototyping into rapid manufacturing applications $[1,2]$. The process has seen a wide variety of alloy processing such as steels, titanium (Ti), aluminum (Al), and magnesium (Mg) alloys. An advantage emerges over electron beam melting (EBM) since thermal conditions during the latter limits its use to high melting temperature materials like $\mathrm{Ti}-6 \mathrm{Al}-4 \mathrm{~V}$ or $\mathrm{Ti}-\mathrm{Al}$ intermetallic [2].

Edwards and Ramulu found fatigue strength of as-built SLM Ti-6Al-4V inferior to the wrought alloy due to porosity and residual stresses [3]. Cain et al. reported that mechanical properties have a significant directional anisotropy. The observation was attributed to anisotropic microstructure with a strong gradient in the cooling direction. Effect of residual stress distribution in the asbuilt specimens was most significant in the fatigue crack growth rate and fracture toughness. Relaxation of residual stresses by post-process annealing reduced anisotropy and increased fracture toughness [1].

Kasperovich and Hausmann concluded that application of optimized scanning parameters with adequate energy density enhanced relative density which improved mechanical properties accordingly. The tensile strength of SLM Ti-6Al-4V in as-built condition was better than the wrought alloy while fracture strain was reduced. In comparison to hot-isostatic pressing (HIP), stress relief (SR) did not enhance high-cycle fatigue (HCF) strength since failure initiated from internal defects which persist after the treatment. In addition to the elimination of defects by HIP, ductility was recovered up to $19.4 \cdot 10^{-2}$ [4]. Wauthle et al. confirmed the same finding concerning HIP for lattice Ti-6Al-4V structures [5].

Leuders et al. reported a prolonged crack initiation phase in SLM Ti-6Al-4V, when porosity is relatively less, in HCF regime. The microstructure which is observed to be dominant $\alpha$ ' increased crack initiation resistance signifycantly [6]. Wycisk et al. found that shot peening reduced fatigue limit by $15 \%$ since it subjected internal porosity to local tensile stresses. In $\mathrm{HCF}$, the analysis reported failures from pores in the diameter range of $40 \mu \mathrm{m}$ or higher [7]. After HIP, crack initiation at internal defects was no longer found. In completely tensile cyclic loading, crack initiation shifts from the surface to subsurface locations [8]. Linear-elastic fracture mechanics (LEFM) calculated fatigue lifetime reliably when long cracks existed but overestimated the lifetime in case of short cracks [9].

\footnotetext{
* Corresponding author: mustafa.awd@tu-dortmund.de
} 
Brandl et al. attempted improving fatigue strength of AlSi10Mg using platform heating (PH) in the SLM process. The as-built microstructure consisted of an $\mathrm{Al}$ matrix where a Si network of equiaxed cells is embedded. In this study, $\mathrm{PH}$ did not increase fatigue strength until a peak hardening treatment is applied after processing [2]. Siddique et al. introduced micro-computed tomography $(\mu-\mathrm{CT})$ in combination with finite-element modeling (FEM) as means to pre-calculate fatigue lifetime for SLM AlSi12 alloy specimens. The analysis introduced stressconcentration factors for pores as a function of diameter and proximity to the surface. Given this, HIP of AlSi12 was assumed to enhance fatigue strength, but due to microstructure alteration and $\mathrm{Si}$ agglomeration, fatigue strength was significantly reduced [10]. Nevertheless, the fatigue strength of SLM AlSi12 in as-built condition was superior to cast alloy. Application of PH reduced mean fatigue strength as well as standard deviation. Since crack initiation from internal porosity was observed, reduction of porosity by $\mathrm{PH}$ increased fatigue strength reliability [11].

Siddique et al. improvised a stochastic approach for fatigue lifetime calculation based on fracture mechanics and the weakest-link theory. The lifetime calculation was found reasonable in low-cycle fatigue (LCF), but not in $\mathrm{HCF}$. The study found that stress-concentration factors induced by surface roughness are much higher than stresses induced by internal porosity. The first reduced fatigue lifetime by one order of magnitude [12]. Hybrid Al structures with reasonable fatigue strength were possible within a hybrid manufacturing framework. After SR the resulting lifetime was better than that of pure SLM structures. The interface between SLM deposition and wrought alloy suffered from microstructure nonhomogeneity as well as significant residual stresses [13]. Production of components from high-strength Al-Sc alloys by SLM was reported with a tensile strength of $\sim 500 \mathrm{MPa}$. Considering fatigue, the strength was significantly reduced by the existence of significant internal porosity [14]. The latest conclusion envisages that if proper process window is developed, high-strength $\mathrm{Al}$ components for dynamic applications in the aerospace industry by SLM are feasible.

In this study, a novel plot-based Weibull model is presented for fatigue lifetime calculation of $\mathrm{Al}$ alloys. The model relies on real-time measurement and modeling techniques for dynamic loading qualification of SLM Al alloys. For two alloys, AlSi10Mg and AlSi12, the combined techniques are applied to conclude about processing and post-processing procedures during SLM process. The model is assessed by experimental fatigue data.

\section{Materials and methods}

\subsection{Experimental testing}

The specimens employed in this study were manufactured at Fraunhofer IAPT using SLM 250 HL. The maximum laser power of the system is $400 \mathrm{~W}$ based on a fiber laser source. Maximum volume capacity inside the building chamber is $250 \times 250 \times 280 \mathrm{~mm}^{3}$ in $3 \mathrm{D}$ space. An inert atmosphere of argon gas was applied for shielding the melt tracks during build-up which was flowing from the left of the chamber to the right. The function of the shielding gas is to ensure purity of melt pools to prevent the formation of inclusions [11]. The scanning method is designed such that porosity and residual stresses are kept to a minimum. The factorial study is presented in Table 1 . The experimental design aims at investigating the effect of platform heating $(\mathrm{PH})$ at $200{ }^{\circ} \mathrm{C}$ on microstructure and eventually fatigue properties by a joint study of experimental testing and modeling procedure for two common compositions of cast $\mathrm{Al}$ alloys, $\mathrm{AlSi} 10 \mathrm{Mg}$ and AlSi12 respectively.

Table 1. Experimental design.

\begin{tabular}{ccccc}
\hline Batch & A & B & C & D \\
\hline Alloy & AlSi10Mg & AlSi10Mg & AlSi12 & AlSi12 \\
\hline SR & no & no & yes & yes \\
\hline PH & no & yes & no & yes \\
\hline
\end{tabular}

The as-built specimens of batches A and B were scanned in a $\mu-C T$ facility with the experimental setup being explained in detail in [14]. Similar results for batches C and $\mathrm{D}$ are presented in [12]. The relative densities for batches A and B were $99.96 \%$ and $99.94 \%$, respectively. The effect of $\mathrm{PH}$ on reducing the number of pores was consistent with [12], but the relative density of the $\mathrm{PH}$ batch was less which disagrees with the previous observation regarding batches $\mathrm{C}$ and $\mathrm{D}$. The volumetric defect distributions of batches $\mathrm{A}$ and $\mathrm{B}$ are presented in Fig. 1. It is observable that batch A developed defects of bigger volume than batch $\mathrm{B}$ while in the latter the smaller diameter porosity $(<120 \mu \mathrm{m})$ was more.

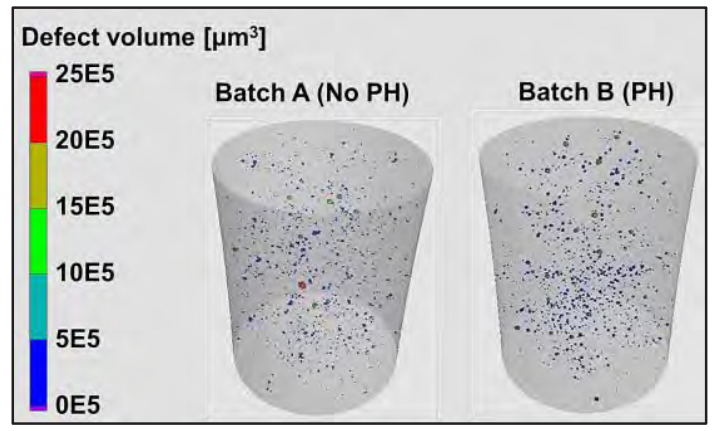

Fig. 1. Volumetric defect distribution from $\mu$-CT of batches A and $\mathrm{B}$.

The relationship between stress developed by porosity and their size was described in detail in [10]. The latter identified pore diameter and location as the most influential factors. The statement renders relative density of such minor difference irrelevant in comparison to the pore distribution within the volume as well as the existence of large critical pores. One more issue that interacts with this factor is the reduction of residual stresses through PH. The latter was also proved to induce microstructure coarsening [11]. The interaction between 
remnant porosity, residual stresses as well as microstructure resulting from $\mathrm{PH}$ is revealed in the mechanical quasi-static and cyclic experiments.

In-situ tensile testing in $\mu-\mathrm{CT}$ was applied, in this study, to be compared with standard ex-situ tensile test for batch D. The aim was to separate porosity and microstructure related mechanisms and the identification of relaxation behavior and crazing effects during plastic deformation. The design of the in-situ experiment inside $\mu$-CT can be envisaged in Fig. 2.

(a)
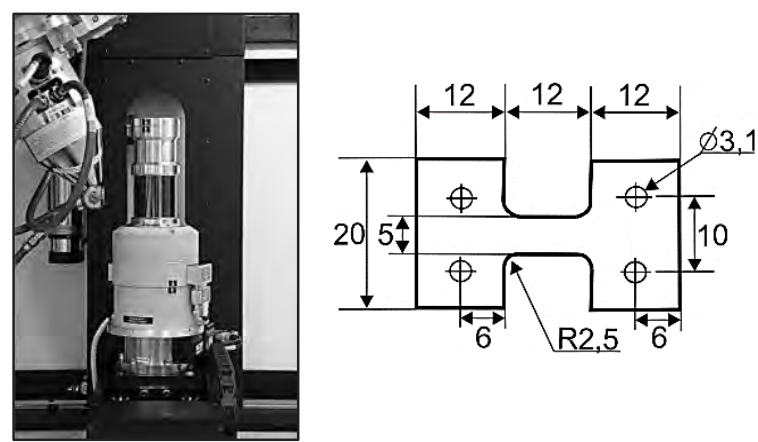

Fig. 2. Design of in-situ $\mu$-CT tensile experiment: (a) mounted in-situ module; (b) specimen geometry.

Quasi-static tensile experiments were carried out on Instron 3369 with a $50 \mathrm{kN}$ load cell using an extensometer with a gauge length of $10 \mathrm{~mm}$. The specimen geometry is shown in Fig. 3a while stroke rate was fixed at $1 \mathrm{~mm} / \mathrm{min}$. The same specimen was used in fatigue experiments at Instron 8872 with $10 \mathrm{kN}$ load cell. The experimental setup of fatigue tests is shown in Fig. 3b. Tensile testing was applied for bridging the relationship between microstructure, defects on one side and fatigue strength on the other through FEM and statistical modeling.

(a)

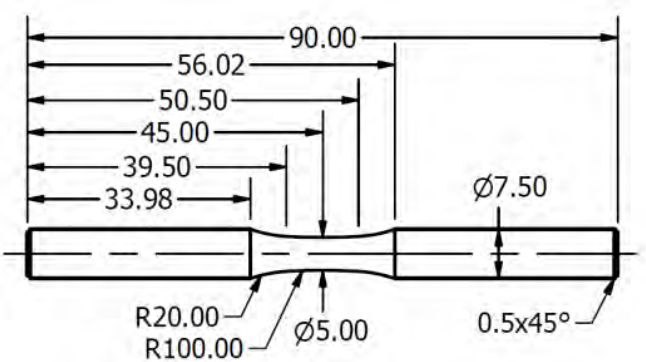

(b)

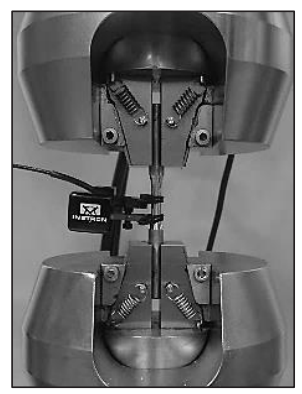

Fig. 3. Design of mechanical testing experiments: (a) specimen geometry; (b) test setup.

\subsection{Modeling and simulation}

Deformation behavior under quasi-static conditions was simulated in this study in Abaqus 6.14. The material law was the true stress-strain curve obtained in a tensile test. The deformation curves of batches $\mathrm{A}$ and $\mathrm{B}$ are shown in Fig. 4. It can be seen that batch B has higher values of Young's modulus, yield strength, ultimate tensile strength and fracture strain. The result agrees with [11] for fracture strain but disagrees with it in ultimate tensile strength. The cited work suggested that microstructure coarsening by $\mathrm{PH}$ increased ductility but reduced tensile strength.

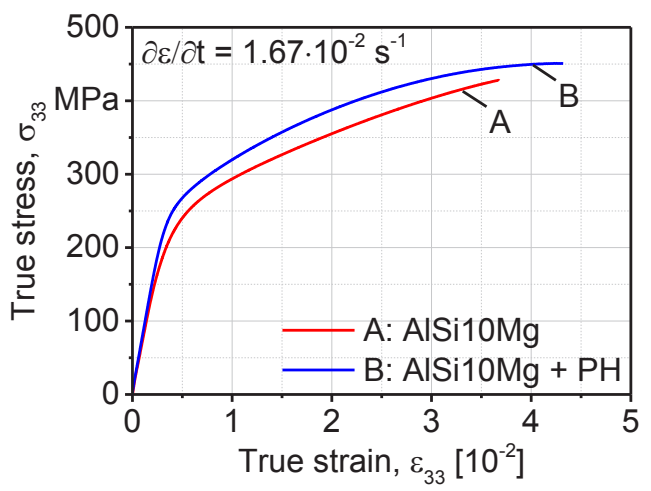

Fig. 4. Flow behavior under quasi-static conditions of batches A and $\mathrm{B}$.

Deformation simulations in Abaqus 6.14 were carried out using the built-in material laws for isotropic and kinematic hardening. A comparison between both material laws as well as the experimental flow curve for batches A and $\mathrm{B}$ can be found in Fig. 5a and Fig. 5b, respectively.

(a)

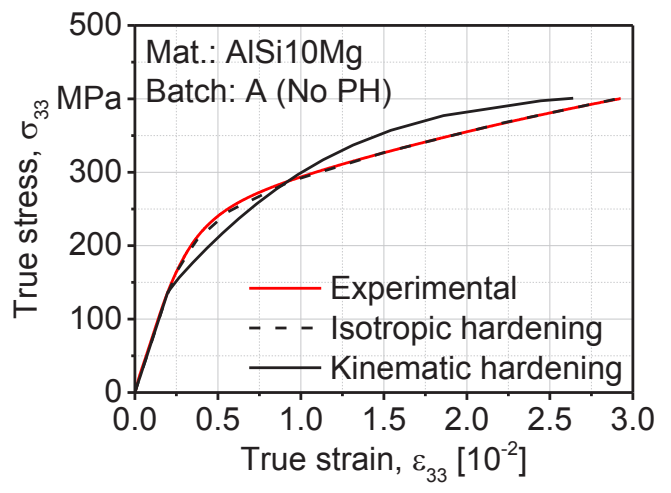

(b)

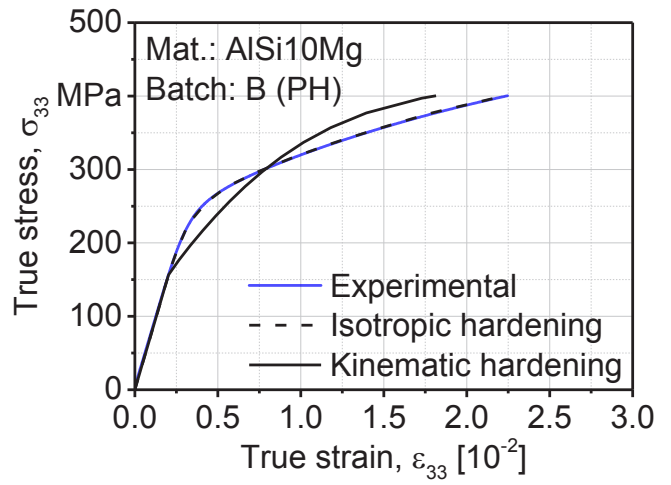

Fig. 5. Comparison between flow curves of the experimental test against simulated conditions under isotropic and kinematic hardening considerations for batches: (a) A (No PH); (b) B (PH). 
The simulation was set to terminate when the crosssectional stress of $400 \mathrm{MPa}$ is developed. The agreement between the experimental flow curves and the isotropic hardening law is good while the discrepancy between both and the kinematic hardening law is clear. Kinematic hardening represents the evolution of peaks of hysteresis under cyclic loading capturing the Bauschinger effect. The finding yields kinematic hardening more representative when modeling fatigue strength of dispersionstrengthened $\mathrm{Al}$ alloys. The conclusion found agreement in $[15]$.

\section{Results and discussion}

\subsection{Mechanical properties}

Siddique et al. reported tensile flow curve of PH AlSi12, here batch $\mathrm{D}$, as seen in Fig. 6 [11]. A comparison between this ex-situ flow behavior against in-situ $\mu$-CT test can be seen in the same figure. During the in-situ test, intermediate $\mu$-CT scans are performed at steps 1-4. Each scan takes $\sim 40 \mathrm{~min}$ at which the indicated force is kept acting on the specimen by the controller. The creeping effect on the stress-strain relation is clear in the figure. The result suggests that cold straining can enhance ductility of the microstructure at the expense of maximum strength. A by-product is to study relaxations under constant static loading as well as deformation of pores and crazing effects.

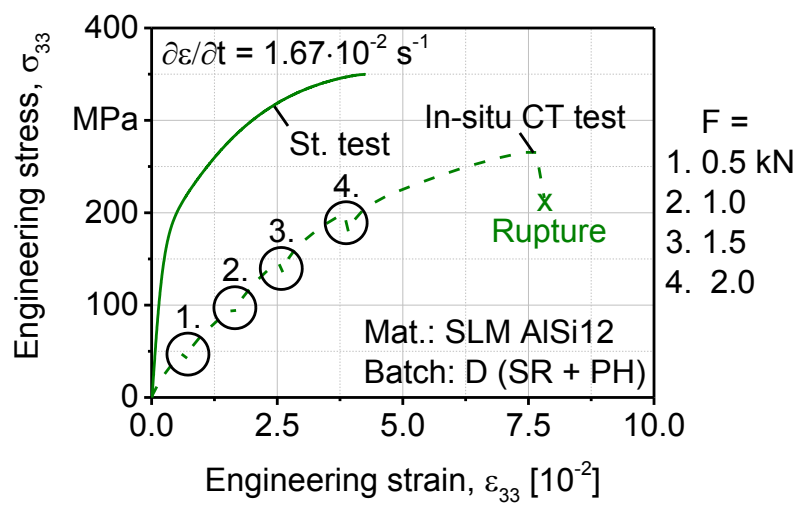

Fig. 6. Comparison between flow curves of the standard ex-situ tensile test and the in-situ $\mu$-CT tensile test.

In comparison to quasi-static tensile test shown in Fig. 4, the comparison between cyclic properties in a continuous load increase test (LIT) is presented in Fig. 7. The principle of this test is explained in detail in [14]. Regarding ultimate tensile strength, batch B was stronger by $\sim 4.65 \%$ in addition to better elasticity modulus. The difference was more pronounced in the cyclic properties since fracture stress was $\sim 37.5 \%$ higher in favor of batch B. This promotes the assumption that micro- and macrostructure of this batch under $\mathrm{PH}$ was significantly tolerant to cyclic damage. Towards $\sim 110 \mathrm{MPa}$, the damage accumulation profile was similar. Afterward, the damage profile of batch A stabilizes on a horizontal plateau until fracture at $\sim 160 \mathrm{MPa}$. Otherwise, batch B adopts constant slope of plastic damage until $\sim 170 \mathrm{MPa}$ at which specimen stiffens until fracture.

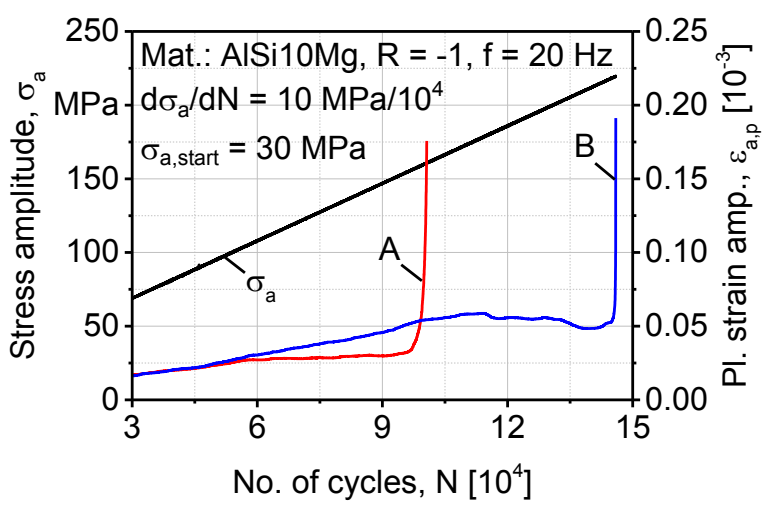

Fig. 7. Plastic strain amplitude reaction in a load increase test for batches: (a) A (No PH); (b) B (PH).

The results of the LIT indicated the response of the plastic damage could vary as a function of the instantaneous stress amplitude. To separate and confirm the mechanism, constant amplitude testing was applied for batches A and $\mathrm{B}$ at the critical stress ranges identified in Fig. 7. As shown in Fig. 8a that under $120 \mathrm{MPa}$ specimen stiffens dynamically in the first $\sim 500$ cycles of the test until a stable stiffness is reached which persists until the end of the test. The footprint of this response is evident in Fig. 7. At $140 \mathrm{MPa}$, which is close to the fracture stress in Fig.7, the specimen softens from the beginning onwards until fracture.

(a)

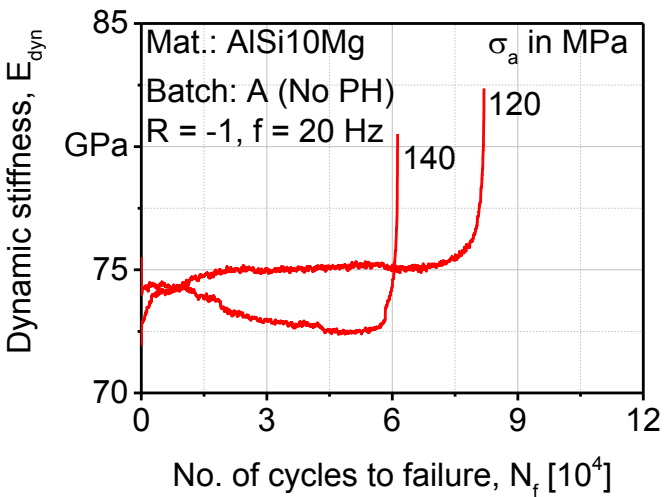

(b)

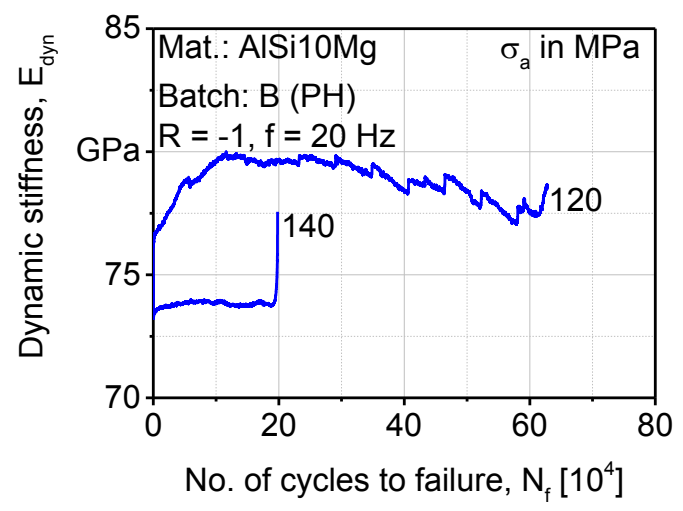

Fig. 8. Evolution of dynamic stiffness under constant amplitude cyclic loading for batches: (a) A (No PH); (b) B (PH).

In Fig. $8 b$, the profile at $120 \mathrm{MPa}$ indicates sharp hardening and increase of dynamic stiffness from the beginning, until $10^{4}$ cycles. Afterward, cycles of softening 
and abrupt hardening develops with overall softening of the specimen. The observation gives evidence about the dynamic healing of the microstructure which resists cyclic damage. The phenomenon is rather attributed to multiscale cyclic damage features with evidence in the microstructure to be found in a later study. At $140 \mathrm{MPa}$, the stiffness has a stable horizon since its near to the transition point in Fig. 7.

Ultrasonic fatigue tests at $20 \mathrm{kHz}(10 \mathrm{kHz}$ effective frequency due to pulse-pause-ratio) were held to identify the effect of $\mathrm{PH}$ on fatigue strength beyond $10^{6}$ cycles. The testing methodology was explained in detail in [13]. In Fig. 9, the corresponding S-N curves for batches $\mathrm{C}$ and $\mathrm{D}$ are reported. Two main observations can be envisaged here. First is that fatigue strength of batch D being 33\% higher and its standard deviation is less in comparison to batch $\mathrm{C}$. The second is consistent with LCF results reported in [11], but the first is not. The observation indicates alteration of damage mechanisms from LCF to VHCF with possibilities to tailor properties specifically for a given fatigue regime. The same analysis is planned for batches $\mathrm{A}$ and $\mathrm{B}$.

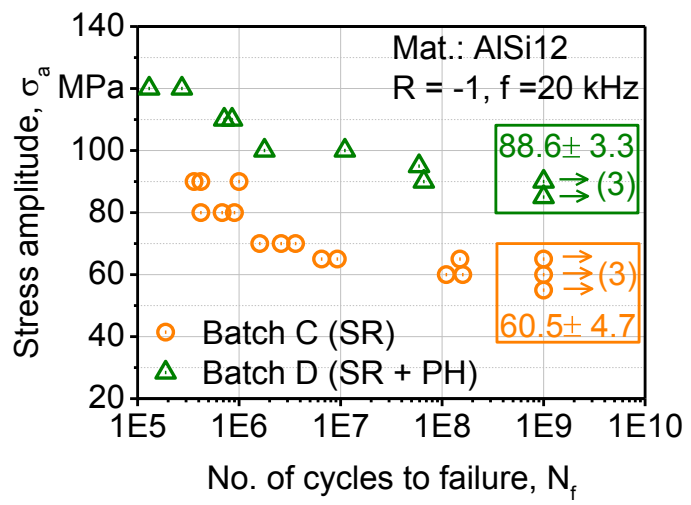

Fig. 9. Very-high-cycle fatigue strength of batches: (a) C (SR + No PH); (b) D (SR + PH).

\subsection{Model-based correlation}

After simulating stress evolution using the kinematic hardening law as shown in Fig. 5, based on $\mu$-CT scans of Fig. 1, FEM results were post-processed in nCode fatigue lifetime calculation software. Element-wise cycles to failure were calculated based on fatigue cut-off criterion. The results were exported to OriginLab data analysis software to plot the Weibull distribution of lifetimes of individual elements. At two stress levels of 140 and $120 \mathrm{MPa}$, the lifetime distribution for batch A can be seen in Fig. 10. It can be seen from Fig.10a to Fig. 10b that the whole plot is shifting to the right as lifetime increases against lower stress amplitudes. The wide distribution of lifetimes from $10^{2}$ to $10^{12}$ cycles is related to local stress state at the element. (a)

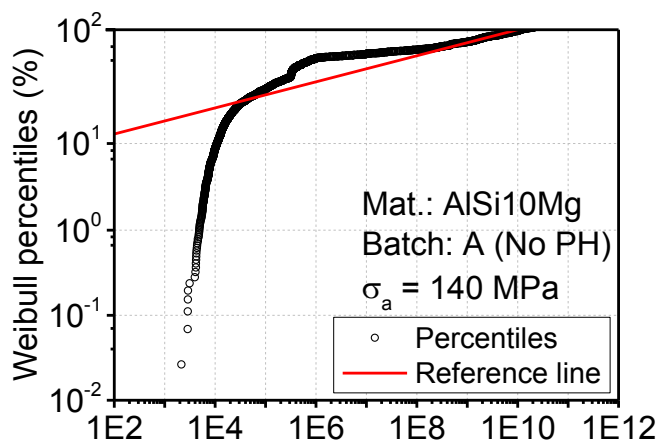

Element-wise no. of cycles to failure, $\mathrm{N}_{f}$

(b)

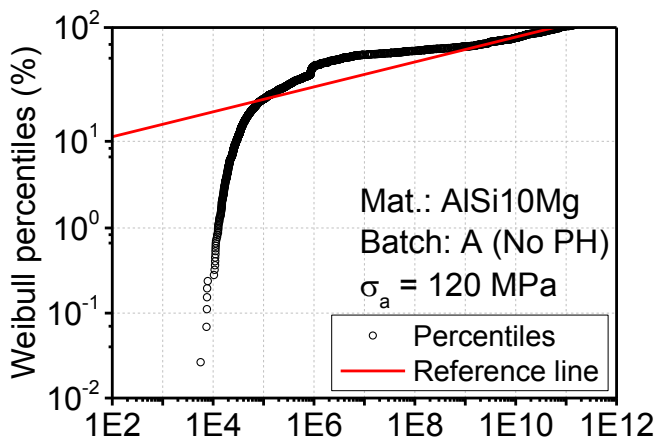

Element-wise no. of cycles to failure, $N_{f}$

Fig. 10. Plot-based Weibull model of fatigue strength of batch A (No PH) at two stress amplitudes (MPa): (a) 140; (b) 120.

As shown in Fig. 10 for batch A and Fig. 11 for batch B, the intersection between Weibull percentiles and the reference line is the mean lifetime of the batch. A onesided confidence interval of $90 \%$ is selected as the lower bound fatigue lifetime criterion. The rule means that the lower bound fatigue lifetime lies at 10\% in Fig. 10 and 11. The upper bound is identified by the first point after the reference line where the slope of Weibull percentiles first becomes infinity. In this manner, fatigue strength characterization is held by identification of a population mean as well as lower and upper bounds as a safe-economic design interval. The approach follows the same concept presented in [14]. The latter was based on Monte-Carlo simulation and the load increase test with extensive computational effort. The approach presented in this work utilizes a flow curve from a tensile test for simulation of kinematic hardening behavior and hysteresis evolution in Abaqus. Post-processing of the element-wise stresses in nCode is followed by plot-based Weibull modeling.

(a)

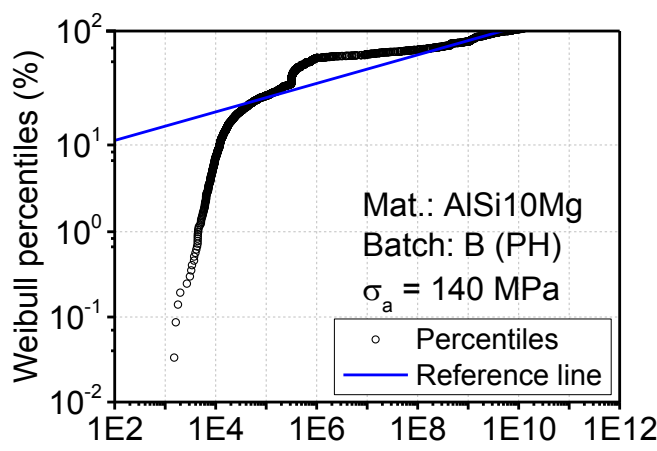

Element-wise no. of cycles to failure, $N_{f}$ 
(b)

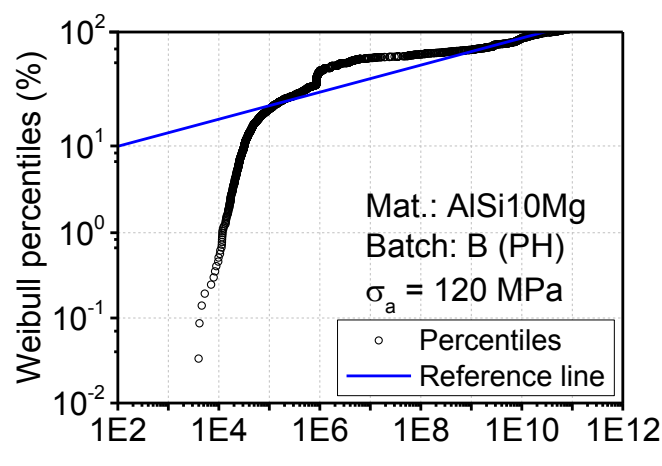

Element-wise no. of cycles to failure, $N_{f}$

Fig. 11. Plot-based Weibull model of fatigue strength of batch B (PH) at two stress amplitudes (MPa): (a) 140; (b) 120.

A comparison between experimental and calculated fatigue lifetime is shown in Fig. 12 for batches A and B. In both batches, the simulation agrees well with experimental data from two perspectives. The first is that mean solid line passes near the mean value of the three validation experiments for each batch and each stress level. The second one is that experimental points are confined to the upper and lower bounds even at experimental points of extreme values. VHCF validation of the predicted lifetimes is planned in later studies. The speed of this procedure is seen as relevant in additive manufacturing since process flexibility allows for tailoring of properties with a wide variety. High-throughput characterization is thus necessary. Selection of proper process parameters to achieve a certain combination of properties with reliability is possible.

(a)

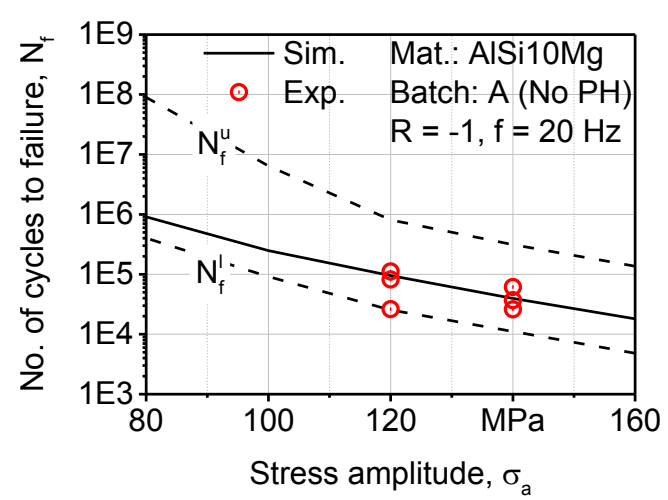

(b)

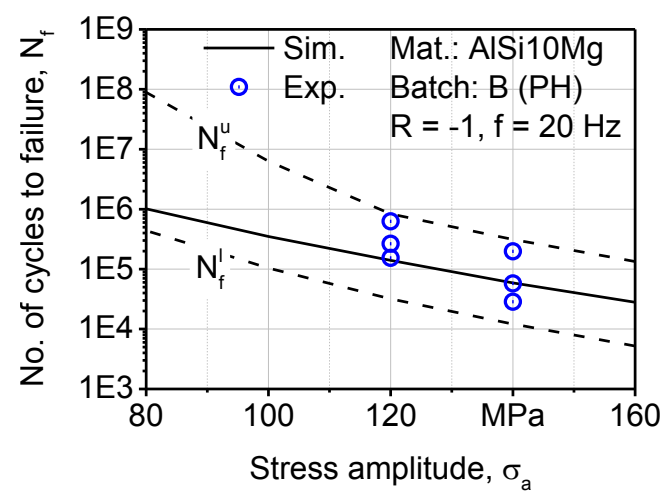

Fig. 12. Comparison between experimental and predicted fatigue strength of batches: (a) A (No PH); B (PH).

\section{Conclusions and outlook}

The current study aimed at investigating efficient testing strategies to qualify additively manufactured aluminum alloys. Two aluminum alloys, AlSi10Mg and AlSi12, were investigated with platform heating as the main factor. The latter factor is identified in the previous literature as a control parameter to reduce remnant porosity and coarsen microstructure $[10,11]$. In this study, it was found consistent that platform heating improved ductility, but tensile strength was not reduced in AlSi10Mg alloy. Consequently, LCF strength of platform heated specimens was better with superiority margin increasing as stress amplitude is reduced. Although the observation contradicts with reported data on the issue, that may be attributed to a change in scanning strategy. This offers an opportunity for a future investigation regarding residual stresses and microstructure control.

In AlSi12 alloy, platform heating improved VHCF strength significantly as determined by ultrasonic fatigue tests. The conclusion needs to be confirmed for AlSi $10 \mathrm{Mg}$ and other additively manufactured alloys. In-situ tensile testing inside $\mu$-CT presented the phenomenon of stress relaxation under constant static load. The phenomenon highlights cyclic creeping that might occur at stress ratios higher than zero. This open question also offers an opportunity for simulation of the effect and probing appropriate material laws to describe it.

The continuous load increase test was used in a previous study to perform an FE simulation of fatigue damage [14]. Based on a demanding computation algorithm of MonteCarlo simulation, fatigue lifetimes were calculated. In this study, the load increase test was used to relate degradation in material's stiffness under constant amplitude to the wide-range fatigue stresses by monitoring plastic strain damage. Development of dynamic stiffness under constant amplitude was consistent with the plastic damage response in a load increase test. It was observed that the stiffness increased asymptotically towards fracture which can be used as failure criterion in a proper fatigue damage modeling scheme.

Built-in kinematic and isotropic hardening material laws of Abaqus were applied to capture cyclic hysteresis evolution and quasi-static deformation, respectively. According to findings of [15] that identified hardening behavior of dispersion-strengthened $\mathrm{Al}$ alloys as kinematic, the resulting flow curve was used to calculate fatigue lifetimes. The latter was computationally efficient as well as reasonably accurate. The result offered designers three levels of design safety by identification of mean population, lower and upper bounds of fatigue lifetime.

The plot-based Weibull model presented here used the probability distribution of element-wise stresses as failure criterion of a macroscale specimen. The finding represents a plasticity-based Weibull model as more accurate and practical in comparison to the approach based on fracture mechanics which was presented in [12]. The VHCF failure mechanism which is agreed to be a 
micromechanical problem can identify in such genre of modeling a potential opportunity to combine a crystal plasticity model with a weakest-link expression for identification of lifetimes required to develop a technical crack beyond microstructurally short ones.

The strategy behind the study is to develop efficiently and accurately presenting models of fatigue lifetime to qualify additively manufactured components of wider potential of tailored properties and functional grading. The field of potential application for the presented methodologies extends to multi-exposure SLM sections and intrinsic heat treatments which are very effective property control tools for allotropic alloys of the Al-Si and Ti-Al alloying systems.

Acknowledgment: The authors would like to thank the German Research Foundation for its financial support to the conduct of this research work in terms of a research grant entitled: "Mechanism-based understanding of functional grading focused on fatigue behavior of additively processed Ti-6Al-4V and Al-12Si alloys" (WA 1672/25-1; EM 95/2-1).

\section{References}

1. Cain, V.; Thijs, L.; van Humbeeck, J.; van Hooreweder, B.; Knutsen, R.: Crack propagation and fracture toughness of Ti6Al4V alloy produced by selective laser melting. Additive Manufacturing (2015) 5: 68-76.

2. Brandl, E.; Heckenberger, U.; Holzinger, V.; Buchbinder, D.: Additive manufactured AlSi10Mg samples using Selective Laser Melting (SLM): Microstructure, high cycle fatigue, and fracture behavior. Materials \& Design (2012) 34: 159-169.

3. Edwards, P.; Ramulu, M.: Fatigue performance evaluation of selective laser melted Ti-6Al-4V. Materials Science and Engineering A (2014) 598: 327-337.

4. Kasperovich, G.; Hausmann, J.: Improvement of fatigue resistance and ductility of TiAl6V4 processed by selective laser melting. Journal of Materials Processing Technology (2015) 220: 202214.

5. Wauthle, R.; Vrancken, B.; Beynaerts, B.; Jorissen, K.; Schrooten, J.; Kruth, J-P.; van Humbeeck, J.: Effects of build orientation and heat treatment on the microstructure and mechanical properties of selective laser melted Ti6Al4V lattice structures. Additive Manufacturing (2015) 5: 77-84.

6. Leuders, S.; Thöne, M.; Riemer, A.; Niendorf, T.; Tröster, T.; Richard, H.; Maier, H.: On the mechanical behavior of titanium alloy TiAl6V4 manufactured by selective laser melting: Fatigue resistance and crack growth performance. International Journal of Fatigue (2013) 48: 300-307.

7. Wycisk, E.; Emmelmann, C.; Siddique, S.; Walther, F.: High cycle fatigue (HCF) performance of Ti-
$6 \mathrm{Al}-4 \mathrm{~V}$ alloy processed by selective laser melting. Advanced Materials Research (2013) 816-817: 134139.

8. Wycisk, E.; Siddique, S.; Herzog, D.; Walther, F.; Emmelmann, C.: Fatigue performance of laser additive manufacture $\mathrm{Ti}-6 \mathrm{Al}-4 \mathrm{~V}$ in very high cycle fatigue regime up to $10^{9}$ cycles. Frontiers in Materials (2015) 2: 2117.

9. Wycisk, E.; Solbach, A.; Siddique, S.; Herzog, D.; Walther, F.; Emmelmann, C.: Effects of defects in laser additive manufactured Ti-6Al-4V on fatigue properties. Physics Procedia (2014) 56: 371-378.

10. Siddique, S.; Imran, M.; Rauer, M.; Kaloudis, M.; Wycisk, E.; Emmelmann, C.; Walther F.: Computed tomography for characterization of fatigue performance of selective laser melted parts. Materials \& Design (2015) 83: 661-669.

11. Siddique, S.; Imran, M.; Wycisk, E.; Emmelmann, C.; Walther, F.: Influence of process-induced microstructure and imperfections on mechanical properties of AlSi12 processed by selective laser melting. Journal of Materials Processing Technology (2015) 221: 205-213.

12. Siddique, S.; Awd, M.; Tenkamp, J.; Walther, F.: Development of a stochastic approach for fatigue life prediction of AlSi12 alloy processed by selective laser melting. Engineering Failure Analysis (2017) 79: 34-50.

13. Siddique, S.; Awd, M.; Tenkamp, J.; Walther, F.: High and very high cycle fatigue failure mechanisms in selective laser melted aluminum alloys. Journal of Materials Research (2017) 32, 23: 4296-4304.

14. Awd, M.; Tenkamp, J.; Hirtler, M.; Siddique, S.; Bambach, M.; Walther, F.: Comparison of microstructure and mechanical properties of Scalmalloy ${ }^{\circledR}$ produced by selective laser melting and laser metal deposition. Materials (2018) 11, 1: $1-17$.

15. Reynolds, A.; Lyons, J.: Isotropic and kinematic hardening in a dispersion-strengthened aluminum alloy. Metallurgical and Materials Transactions A (1997) 28, 5 : 1205-1211. 